\title{
Endoscopic Therapy for Pancreatic Fluid Collections: A Definitive Management Using a Dedicated Algorithm
}

\author{
Ming Ming Xu', Iman Andalib², Aleksey Novikov ${ }^{3}$, Enad Dawod ${ }^{4}$, Moamen Gabr ${ }^{4}$, Monica Gaidhane ${ }^{2}$, Amy Tyberg ${ }^{2}$ and \\ Michel Kahaleh ${ }^{2}$ \\ Division of Gastroenterology, 'Southern California Permanente Medical Group, Los Angeles, CA, ${ }^{2}$ Rutgers University, Robert Wood Johnson \\ Medical School, New Brunswick, NJ, ${ }^{3}$ Thomas Jefferson University Hospital, Philadelphia, PA, ${ }^{4}$ Weill Cornell Medical College, New York, NY, \\ USA
}

Background/Aims: Endoscopic ultrasonography (EUS)-guided drainage is the preferred approach for infected or symptomatic pancreatic fluid collections (PFCs). Here, we developed an algorithm for the management of pancreatitis complicated by PFCs and report on its efficacy and safety.

Methods: Between September 2011 and October 2017, patients were prospectively managed according to the algorithm. PFCs were classified as poorly organized fluid collections (POFCs), pancreatic pseudocysts (PPs), or walled-off pancreatic necrosis (WOPN). Clinical success was defined as a decrease in PFC size by $\geq 50 \%$ of the maximal diameter or to $\leq 2 \mathrm{~cm}$.

Results: A total of 108 patients (62\% male; mean age, 53 years) were included: 13 had POFCs, 43 had PPs, and 52 had WOPN. Seventytwo patients (66\%) required a pancreatic duct (PD) stent, whereas $65(60 \%)$ received enteral feeding. A total of 103 (95\%) patients achieved clinical success. Eight patients experienced complications including bleeding $(n=6)$ and surgical intervention $(n=2)$. Patients with enteral feeding were 3.4 times more likely to achieve resolution within 60 days $(p=0.0421)$, whereas those with PD stenting was five times more likely to achieve resolution within 90 days $(p=0.0069)$.

Conclusions: A high PFC resolution rate can be achieved when a dedicated algorithm encompassing EUS-guided drainage, PD stenting, and early enteral feeding is adopted. Clin Endosc 2020;53:355-360

Key Words: Endoscopic ultrasonography; Enteral feeding; Pancreatic pseudocyst; Pancreatitis; Stents

\section{INTRODUCTION}

Pancreatic fluid collections (PFCs) may result from pancreatic trauma, acute and chronic pancreatitis, and pancreatic surgery. ${ }^{1,2}$ Based on the 2008 revised Atlanta International Symposium on Acute Pancreatitis, PFCs are characterized as acute peri-PFCs, acute necrotic collections (ANCs), pancreatic pseudocysts (PPs), and walled-off pancreatic necrosis

Received: June 6, 2019 Revised: July 3, 2019

Accepted: July 3, 2019

Correspondence: Michel Kahaleh

Division of Gastroenterology, Rutgers University, Robert Wood Johnson Medical School, 1 RWJ Plaza, MEB 464, New Brunswick, NJ 08901, USA

Tel: +1-732-235-7784, Fax: +1-732-235-7784, E-mail: mkahaleh@gmail.com ORCID: https://orcid.org/0000-0003-0836-6114

(c) This is an Open Access article distributed under the terms of the Creative Commons Attribution Non-Commercial License (http://creativecommons.org/ licenses/by-nc/3.0) which permits unrestricted non-commercial use, distribution, and reproduction in any medium, provided the original work is properly cited.
(WOPN). ${ }^{2-4}$ This classification distinguishes PFCs to guide their management. PPs are encapsulated collections of amylase-rich fluid with a well-defined inflammatory wall but no necrotic material that usually result from acute peri-PFCs after 4 weeks, while WOPN is a mature, encapsulated collection of pancreatic and/or peripancreatic necrosis that usually occur 4 weeks after an acute episode of necrotizing pancreatitis. ${ }^{2}$ These collections differ from acute peri-PFCs and ANCs, which are considered poorly organized fluid collections (POFCs) and might resolve with conservative management. ${ }^{5}$ The diagnosis is currently made by imaging modalities such as computed tomography (CT), magnetic resonance imaging (MRI), ${ }^{6}$ or endoscopic ultrasonography (EUS). ${ }^{7}$

Indications for and the timing of drainage of PFCs have been controversial in recent years. ${ }^{8,9}$ Most POFCs resolve spontaneously without intervention. However, PPs and WOPN usually require intervention, and drainage is required 
if cause symptoms such as pain, infection, or gastric or biliary obstruction. Historically, surgery has been the primary drainage method. However, with recent advances in therapeutic EUS, endoscopic approaches to drainage have become the preferable approach, with fewer adverse events, shorter hospital stays, and similar efficacy. ${ }^{10-13}$

Pancreatic duct (PD) disruption has been associated with an increased risk of recurrence of PFCs, decreased rate of PFC resolution after drainage, and recurrent pancreatitis. ${ }^{3,14-19}$ Identifying a PD injury via secretin-magnetic resonance cholangiopancreatography (MRCP) or endoscopic retrograde cholangiopancreatography (ERCP) is important for treatment success. Finally, the use of early nutritional support has also been part of the management of acute pancreatitis. Its benefits have been also evaluated extensively in the treatment of severe pancreatitis and PFCs with supporting results. ${ }^{3,20}$

At our institution, patients with necrotizing pancreatitis are treated with a dedicated algorithm combining early enteral feeding, endoscopic drainage of PP and WOPN with endoscopic necrosectomy as needed, and PD stenting in cases of suspected PD leak or injury. We aimed to assess the efficacy and safety of this algorithm for managing pancreatitis with PFCs.

\section{MATERIALS AND METHODS}

Consecutive patients who presented with acute pancreatitis complicated by the development of symptomatic PFCs between September 2011 and October 2017 were prospectively enrolled in a dedicated registry on November 8, 2011,
(ClinicalTrials.gov identifier: NCT01522573) and managed according to the proposed algorithm (Fig. 1). All PFCs were classified as POFCs, PPs, or WOPN. We incorporated all acute peri-PFCs and ANCs into the POFC category.

\section{Procedure}

All PPs and WOPN underwent EUS-guided drainage with stent placement. In WOPN, a lumen-apposing metal stent (LAMS) or large-diameter covered esophageal metal stent (FCEMS) was placed to permit direct endoscopic necrosectomy (DEN). LAMSs (Axios; Boston Scientific, Natick, MA, USA) were 10, 15, or $20 \mathrm{~mm}$ in diameter. Fully covered self-expanding metal stents (FCSEMS; Taewoong, Gimpo, Korea) were 18 - or $20 \mathrm{~mm}$ in diameter and $60 \mathrm{~mm}$ in length. Using EUS, the collection was punctured with a 19-gauge fine needle aspiration (FNA) needle through the gastric or duodenal wall. A guidewire was advanced through the FNA needle, and the tip was coiled deeply into the collection under EUS and fluoroscopic guidance. The FNA needle was then retracted from the puncture site, leaving the wire in position. The cystenterotomy tract was then created using cautery-enhanced LAMS or a 10-Fr cystotome (Wilson Cook, Winston-Salem, NC, USA). Subsequently, an FCEMS or LAMS was placed to allow mechanical debridement of the necrotic tissue under direct endoscopy-guided visualization. In all DEN cases, hydrogen peroxide in a 1:5 dilution with normal saline was used to help dissolve the necrotic debris, dislodge the necrotic tissue, and extract the debris. The procedure was performed in multiple sessions until all necrotic debris was completely removed as assessed endoscopically and/or by supporting cross-sectional imaging showing resolution of the PFC.

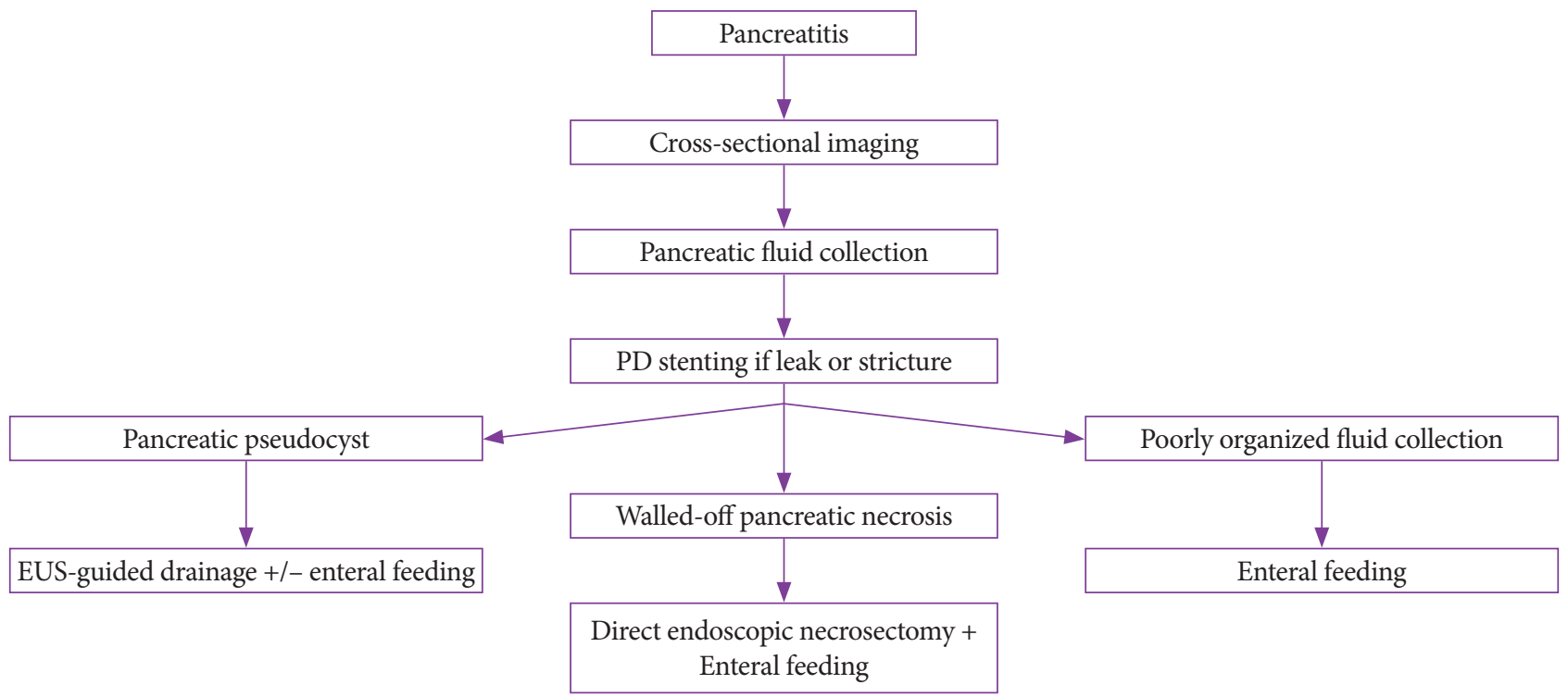

Fig. 1. Algorithm for endoscopic therapy. EUS, endoscopic ultrasonography; PD, pancreatic duct. 
Early enteral nutrition via a nasojejunal feeding tube or a percutaneous gastrojejunostomy tube was initiated in all cases of PFCs with severe pancreatitis. Severe pancreatitis was defined by the CT severity index. ${ }^{20}$ No patients required total parenteral nutrition (TPN).

ERCP with pancreatic cannulation was performed in patients with clinically suspected PD injury prior to drainage of the PFCs. Pancreatic sphincterotomy with the placement of a PD stent was performed in patients with PD strictures or disruption. A biliary sphincterotomy was offered to patients with gallstone pancreatitis.

\section{Definitions}

The primary outcome was clinical success defined as a decrease in PFC size by $\geq 50 \%$ of maximal diameter or to $\leq 2 \mathrm{~cm}$ together with resolution of symptoms. The secondary outcome was the complete resolution of PFCs, defined as no imaging or endoscopic evidence of residual or recurrent PFCs together with symptom resolution after stent removal over the follow-up period. Adverse events were defined as bleeding requiring transfusion, percutaneous embolization, or repeat endoscopic intervention, postoperative infection, bowel ischemia, or perforation requiring surgical intervention.

\section{Follow-up}

Follow-up was performed on a monthly basis with cross-sectional imaging until clinical success was achieved, at which point the cystenterotomy stent was removed. The evaluation of continued resolution or recurrence of the $\mathrm{PFC}$ was documented by cross-sectional imaging CT or MRI until up to 6 months post-stent removal.

\section{Statistical analysis}

All statistical analyses were performed using SPSS 15.0 statistics software (SPSS Inc., Chicago, IL, USA). A $t$-test for two dependent means and further logistical regression analysis were performed to assess for independent predictors of the resolution of PFC.

\section{RESULTS}

A total of 108 consecutive patients with acute pancreatitis who developed symptomatic PFCs were entered into a dedicated prospective database (Table 1). The mean age of the cohort was 51.8 (standard deviation [SD], 16.3) years; 61 subjects were male (61\%). The etiology of pancreatitis was gallstone-related $(n=42[38 \%])$, alcohol-related $(n=19$ [17.5\%]), post-surgical ( $n=13[12 \%])$, pancreas divisum $(n=6[5.5 \%])$, hypertriglyceridemia $(n=5[5 \%])$, post-ERCP pancreatitis $(n=7$
[6.5\%]), idiopathic ( $n=5[5 \%])$, and other $(n=11$ [10\%]; autoimmune pancreatitis, medication-induced, hereditary pancreatitis, traumatic or pancreatic cancer induced) (Table 1). Of the 108 patients with PFCs, 13 (12\%) had POFCs, 43 (40\%) had PPs, and 52 (48\%) had WOPN. Patients with symptomatic PPs or WOPN $(n=95)$, defined as clinical signs of an infected PFC (leukocytosis, fever, sepsis), pain, or early satiety due to gastric compression, underwent endoscopic drainage. Drainage was performed using LAMS (55), FCSEMS (34), or plastic (5). The stent choice was dependent on the complexity of the fluid collection as well as stent availability (LAMS became available at our institution in 2014, at which time it was the preferred stent of choice for complex fluid collection until FCSEMS were

Table 1. Clinical Characteristics of Patients

\begin{tabular}{|c|c|c|}
\hline Characteristics & Total $(n=108)$ & $p$-values \\
\hline Age (range, SD) & 51.8 yr $(14-90,16.3)$ & \\
\hline Gender, Male (\%) & $67 / 108(62 \%)$ & \\
\hline \multicolumn{3}{|l|}{ PFC groups (\%) } \\
\hline $\begin{array}{l}\text { Poorly organized fluid } \\
\text { collection }\end{array}$ & $13 / 108(12 \%)$ & \\
\hline Pancreatic pseudocyst & 43/108 (40\%) & \\
\hline $\begin{array}{l}\text { Walled-off pancreatic } \\
\text { necrosis }\end{array}$ & $52 / 108(48 \%)$ & \\
\hline \multicolumn{3}{|l|}{ Etiology } \\
\hline Autoimmune & $2 / 108$ & \\
\hline Medication induced & $4 / 108$ & \\
\hline Pancreas divisum & $6 / 108$ & \\
\hline $\begin{array}{l}\text { Alcohol induced } \\
\text { pancreatitis }\end{array}$ & $19 / 108$ & \\
\hline $\begin{array}{l}\text { Gallbladder stones } \\
\text { induced pancreatitis }\end{array}$ & $42 / 108$ & \\
\hline Hereditary & $1 / 108$ & \\
\hline Hypertriglyceridemia & $5 / 108$ & \\
\hline Idiopathic & $5 / 108$ & \\
\hline Post surgical & $13 / 108$ & \\
\hline Post ERCP pancreatitis & $7 / 108$ & \\
\hline Cancer related & $3 / 108$ & \\
\hline Trauma & $1 / 108$ & \\
\hline Size of PFC pre-treatment & $9.2 \mathrm{~cm}(1.8-29.8)$ & $p<0.0001$ \\
\hline $\begin{array}{l}\text { Size of PFC post } \\
\text { treatment }\end{array}$ & $0.89 \mathrm{~cm}(0-10)$ & $\begin{array}{c}\text { Mean decrease } \\
\text { of }-8.66 \mathrm{~cm}\end{array}$ \\
\hline Recurrence rate (\%) & 9/108 (8.3\%) & \\
\hline Mortality rate (\%) & $4 / 108(3.7 \%)$ & \\
\hline Multiorgan failure & $3 / 4$ & \\
\hline
\end{tabular}

ERCP, endoscopic retrograde cholangiopancreatography; PFC, pancreatic fluid collection; SD, standard deviation. 
used). The majority of patients underwent transgastric drainage $(n=87$ [80.5\%]). Drainage location was chosen based on the optimal location for EUS-guided drainage. Nine patients required percutaneous drainage of the pelvic collections. All patients with WOPN underwent a mean four sessions (range, 1-16) of DEN. The mean PFC size prior to any treatment was 9.3 (SD, 5.5) $\mathrm{cm}$ as determined by cross-sectional imaging, which decreased to mean $0.72(\mathrm{SD}, 1.8) \mathrm{cm}$ post-drainage $(p<0.01)$

A total of 74 patients $(68.5 \%)$ underwent a concurrent ERCP for the evaluation of suspected PD injury or stricture and PD stenting for treatment of PD leak; 19 (25.6\%) had a temporary PD stent placed for prophylaxis; and 2 (3\%) had failed PD cannulation due to severe duodenal inflammation. Those patients who did not undergo ERCP had no clinical suspicion for PD injury on MRCP.

For long-term nutritional support, 65 patients (60\%) required enteral nutrition: five (8\%) via nasojejunal feeding tube and $60(92 \%)$ via a percutaneous gastrojejunostomy tube.

Clinical success was achieved in 103 of 105 patients (95\%). POFCs were resolved in all $13(100 \%)$ patients, PPs in all 42 (100\%) patients, and WOPN in 48 of 52 (92\%) patients (Table 2).

Finally, multivariate logistic regression analysis was performed to assess for independent predictors of PFC resolution within 60 or 90 days. After controlling for PD leak, PD stenting, enteral nutrition use, and PFC subtype, EUS-guided drainage remained a significant predictor of resolution of the PFC (odds ratio [OR], 21.9; 95\% confidence interval [CI], 1.7276.0; $p=0.017$ ). After adjusting for the presence of PD leak, PFC type, necrosectomy, and PD stenting, enteral feeding was
3.4 times (OR, 3.4; 95\% CI, 1-10.9) more likely to achieve resolution within 60 days $(p=0.0421)$.

After adjusting for presence of PD leak, PFC type, necrosectomy, and enteral feeding, PD stenting was almost five times (OR, 4.8; 95\% CI, 1.5-12.2) more likely to achieve resolution within 90 days $(p=0.0069)$.

Patients were followed for at least 6 months post-resolution (mean follow-up, 17.5 months). PFC recurrence occurred in 9 of 100 (9\%) of patients in the follow-up period. Adverse events were seen in eight patients and included bleeding $(n=6$; four requiring percutaneous embolization, two managed endoscopically) and surgical intervention ( $n=2$; one bowel necrosis, one entero-cutaneous fistula). Three patients died of multi-organ failure secondary to the underlying pancreatitis but unrelated to the procedures, while one other died of a bleeding pseudoaneurysm.

\section{DISCUSSION}

Endoscopic cystenterotomy has become the preferred management method for PFCs. However, the overall success rate for PFC treatment varies in the literature from $82 \%$ to $100 \%$ of cases, with complications occurring in 5\% to $16 \%$ and recurrence rates up to $19 \%{ }^{19-28}$ This discrepancy in the literature is probably related to the absence of a dedicated algorithm addressing not only the PFC but evaluating PD injury and concurrent nutrition support. Here we evaluated our novel algorithm for necrotizing pancreatitis using early enteral nutrition for severe pancreatitis, endoscopic drainage of PP and

Table 2. Clinical Characteristics Categorized by Type of Fluid Collection

\begin{tabular}{|c|c|c|c|}
\hline Type of fluid collection & $\begin{array}{c}\text { Poorly organized fluid } \\
\text { collection } \\
n=13\end{array}$ & $\begin{array}{l}\text { Pancreatic pseudocyst } \\
\qquad n=43\end{array}$ & $\begin{array}{c}\text { Walled-off pancreatic } \\
\text { necrosis } \\
n=52\end{array}$ \\
\hline Pancreatic duct leak present & $5(38 \%)$ & $24(56 \%)$ & $31(60 \%)$ \\
\hline Enteral feeding conducted & $6(46 \%)$ & $14(33 \%)$ & $45(86.5 \%)$ \\
\hline Pancreatic duct stenting done & $11(85 \%)$ & $28(65 \%)$ & $35(67 \%)$ \\
\hline Resolution rate & $13(100 \%)$ & $42(100 \%)$ & $48(92 \%)$ \\
\hline \multicolumn{4}{|l|}{ Adverse events } \\
\hline Pseudoaneurysm bleed & & $2(5 \%)$ & \\
\hline \multicolumn{4}{|l|}{ Bleeding needing IR embolization } \\
\hline Gastrointestinal bleed at cystgastrostomy site & & $1(2 \%)$ & $3(6 \%)$ \\
\hline Pancreatic fluid collection & & & $1(2 \%)$ \\
\hline Enterocutaneous fistula & & & $1(2 \%)$ \\
\hline Stent migration & & & $1(2 \%)$ \\
\hline Bowel perforation & & & $1(2 \%)$ \\
\hline
\end{tabular}

IR, interventional radiology. 
WOPN with endoscopic necrosectomy as needed, and PD stenting in cases of suspected PD leak or injury.

Multiple endoscopic approaches have been created for draining PFCs. Transmural drainage is performed by accessing the collection by creating a tract through the gastric or duodenal wall and placing one or multiple stents. This procedure was traditionally performed using double-pigtail plastic stents; however, recently FCSEMS or novel lumen-apposing covered self-expandable metal stents have also been used with similar results. ${ }^{2}$ Traditionally, PFCs were drained under endoscopic visualization of a visible indentation of the gastric or duodenal wall by the bulging PFC; however, it is estimated that in about $42 \%$ of cases, there is no bulging impression, which limits the efficacy and safety of this technique. ${ }^{29}$ With the evolution and refinement of therapeutic EUS techniques, much higher success rates have been reported with EUS-guided drainage of PFC. ${ }^{27-29}$ In our study, all patients underwent EUS-guided drainage with stent placement. A recent systematic review of 17 studies comparing the use of plastic and metal stents in transmural drainage found no significant difference in the drainage complication rates (16\% [ $95 \%$ CI, $14 \%-39 \%$ ] vs. $23 \%$ [95\% CI, 16\%-33\%]]..$^{30}$ Furthermore, the rate of adverse events appears to be dependent on the type of collection drained, not the particular stent used, with a higher incidence of adverse events seen in WOPN, ${ }^{10,18}$ which is also consistent with our findings in which 7 of 10 adverse events occurred in patients with WOPN.

In recent years, DEN has been used to treat these collections. Using EUS, the collection can be accessed through the gastric or duodenal wall using a tract. Subsequently, the tract is dilated to allow the debridement of necrotic tissues under direct endoscopy visualization. Debridement may be required every 48-72 hours until complete removal of all necrotic debris is achieved. ${ }^{31-33}$ In our study, all patients with WOPN underwent a mean 4 (range, 1-16) DEN sessions, similar to those in other studies. In a multicenter study of 93 patients undergoing DEN, Seifert et al. showed $80 \%$ clinical success rate, with a $23 \%$ complication rate and $7.5 \%$ mortality rate. ${ }^{34}$ With the development of LAMS, EUS-guided drainage of WOPN has been become even more streamlined. In 2015, a prospective multicenter study evaluated 46 patients with WOPN who underwent EUS-guided drainage with LAMS stents, showing a clinical success rate of $81 \%$ and an overall major complications rate of $9 \%$ due to infection from stent occlusion. ${ }^{35}$ The safety and efficacy of these stents have been evaluated in other studies with similar conclusions. ${ }^{3}$

PD disruption has been associated with an increased risk of PFC recurrence and a decreased rate of PFC resolution after drainage. ${ }^{3,14-19}$ Thus, evaluating PD for any disruption via MRCP or ERCP plays a crucial role in treatment success. In
2009, Nealon et al. demonstrated that PD disruption decreased the rate of spontaneous resolution of PPs ( $0 \%-5 \%$ compared with $87 \%$ in patients with a normal PD). ${ }^{16}$ They also showed that patients with necrotizing pancreatitis who had ductal changes were most likely to have immediate and delayed complications. ${ }^{16}$ Furthermore, in 2010, Trevino et al. showed improved PFC resolution in patients who underwent PFC drainage with PD stenting (97.5\%) compared to those who underwent PFC drainage alone (80\%). ${ }^{2,17}$ In our study, prior to $\mathrm{PD}$ exploration, all patients underwent MRCP. If there was clinical suspicion of a PD injury, they underwent ERCP for the evaluation of PD integrity. In addition, 53 (49\%) underwent PD stent placement for therapy, 19 (17.6\%) underwent prophylactic PD stent placement, and 2 (3\%) experienced PD cannulation failure and were managed strictly with enteral feeding after PFC drainage.

Lastly, the use of early nutritional support has been an integral part in the management of necrotizing pancreatitis for almost two decades. In 2010, Al-Omran et al. published a meta-analysis comparing randomized trials of enteral nutrition vs. TPN in severe pancreatitis and showed that enteral feeding was associated with a significant reduction in mortality and systemic infections. ${ }^{23}$ Therefore, enteral feeding has become the standard of care for nutritional support in patients with acute pancreatitis. In addition, the early initiation of enteral nutrition has been evaluated extensively in the treatment of PFCs with supporting results. ${ }^{3}$ In 2013, Li et al. showed that patients who received early initiation of enteral nutrition (within 48 hours of admission) had significantly lower complication rates. ${ }^{21}$ In our study, 65 patients $(60 \%)$ with severe pancreatitis received enteral nutrition, of whom, 60 (92\%) underwent percutaneous gastrojejunostomy placement.

Four patients with more severe disease and large infected fluid collections did not respond to this algorithm. This is probably related to their comorbidities (diabetes, obesity, cirrhosis) and late referral without appropriate aggressive early management, such as hydration and enteral nutrition.

In conclusion, endoscopic drainage has become the preferable approach for the management of these collections, with fewer adverse events, shorter hospital stays, and similar efficacy. ${ }^{10,11}$ The successful resolution of PFCs can be achieved within 2-3 months of intervention when a dedicated algorithm is adopted that includes EUS-guided cystogastrostomy, PD exploration with stenting, and early enteral feeding.

\section{Conflicts of Interest}

Michel Kahaleh received grant support from Boston Scientific, Fujinon, EMcison, Xlumena Inc., W.L. Gore, MaunaKea, Apollo Endosurgery, Cook Endoscopy, ASPIRE Bariatrics, GI Dynamics, NinePoint Medical, Merit Medical, Olympus, and MI Tech. He is a consultant for Boston Scientific, Xlumena Inc., Concordia Laboratories Inc, ABBvie, and MaunaKea Tech. 
Amy Tyberg is a consultant for Endogastric Solutions. The other authors have no financial conflicts of interest.

\section{Author Contributions}

Conceptualization: Michel Kahaleh

Data curation: Ming Ming Xu, Iman Andalib, Aleksey Novikov, Enad

Dawod, Moamen Gabr, Monica Gaidhane, Amy Tyberg, MK

Formal analysis: MMX, IA, AN, ED, MGab, MGai, AT, MK

Methodology: MK

Supervision: MK

Validation: MK

Writing-original draft: MMX, IA, AN, ED, MGab, MGai, AT

Writing-review\&editing: MK

\section{REFERENCES}

1. Holt BA, Varadarajulu S. The endoscopic management of pancreatic pseudocysts (with videos). Gastrointest Endosc 2015;81:804-812.

2. ASGE Standards of Practice Committee, Muthusamy VR, Chandrasekhara V, et al. The role of endoscopy in the diagnosis and treatment of inflammatory pancreatic fluid collections. Gastrointest Endosc 2016;83:481-488

3. Tyberg A, Karia K, Gabr M, et al. Management of pancreatic fluid collections: a comprehensive review of the literature. World J Gastroenterol 2016;22:2256-2270.

4. Bergman S, Melvin WS. Operative and nonoperative management of pancreatic pseudocysts. Surg Clin North Am 2007;87:1447-1460, ix.

5. Ge PS, Weizmann M, Watson RR. Pancreatic pseudocysts: advances in endoscopic management. Gastroenterol Clin North Am 2016;45:9-27.

6. Aghdassi A, Mayerle J, Kraft M, Sielenkämper AW, Heidecke CD, Lerch MM. Diagnosis and treatment of pancreatic pseudocysts in chronic pancreatitis. Pancreas 2008;36:105-112.

7. Rana SS, Bhasin DK, Reddy YR, et al. Morphological features of fluid collections on endoscopic ultrasound in acute necrotizing pancreatitis: do they change over time? Ann Gastroenterol 2014;27:258-261.

8. Fisher JM, Gardner TB. Endoscopic therapy of necrotizing pancreatitis and pseudocysts. Gastrointest Endosc Clin N Am 2013;23:787-802.

9. Aghdassi AA, Mayerle J, Kraft M, Sielenkämper AW, Heidecke CD, Lerch MM. Pancreatic pseudocysts--when and how to treat? HPB (Oxford) 2006;8:432-441.

10. Varadarajulu S, Bang JY, Phadnis MA, Christein JD, Wilcox CM. Endoscopic transmural drainage of peripancreatic fluid collections: outcomes and predictors of treatment success in 211 consecutive patients. J Gastrointest Surg 2011;15:2080-2088.

11. Varadarajulu S, Bang JY, Sutton BS, Trevino JM, Christein JD, Wilcox CM. Equal efficacy of endoscopic and surgical cystogastrostomy for pancreatic pseudocyst drainage in a randomized trial. Gastroenterology 2013;145:583-590.e1.

12. van Santvoort HC, Bakker OJ, Bollen TL, et al. A conservative and minimally invasive approach to necrotizing pancreatitis improves outcome. Gastroenterology 2011;141:1254-1263.

13. Warshaw AL, Rattner DW. Timing of surgical drainage for pancreatic pseudocyst. Clinical and chemical criteria. Ann Surg 1985;202:720-724.

14. Neoptolemos JP, London NJ, Carr-Locke DL. Assessment of main pancreatic duct integrity by endoscopic retrograde pancreatography in patients with acute pancreatitis. Br J Surg 1993;80:94-99.

15. Howard TJ, Moore SA, Saxena R, Matthews DE, Schmidt CM, Wiebke EA. Pancreatic duct strictures are a common cause of recurrent pancreatitis after successful management of pancreatic necrosis. Surgery 2004:136:909-916.
16. Nealon WH, Bhutani M, Riall TS, Raju G, Ozkan O, Neilan R. A unifying concept: pancreatic ductal anatomy both predicts and determines the major complications resulting from pancreatitis. J Am Coll Surg 2009;208:790-799; discussion 799-801.

17. Trevino JM, Tamhane A, Varadarajulu S. Successful stenting in ductal disruption favorably impacts treatment outcomes in patients undergoing transmural drainage of peripancreatic fluid collections. J Gastroenterol Hepatol 2010;25:526-531.

18. Lau ST, Simchuk EJ, Kozarek RA, Traverso LW. A pancreatic ductal leak should be sought to direct treatment in patients with acute pancreatitis. Am J Surg 2001;181:411-415.

19. Iso Y, Kubota K. Intragastric stapled pancreatic pseudocystgastrostomy under endoscopic guidance. Surg Laparosc Endosc Percutan Tech 2013;23:330-333.

20. Makola D, Krenitsky J, Parrish C, et al. Efficacy of enteral nutrition for the treatment of pancreatitis using standard enteral formula. Am J Gastroenterol 2006;101:2347-2355.

21. Li JY, Yu T, Chen GC, et al. Enteral nutrition within 48 hours of admission improves clinical outcomes of acute pancreatitis by reducing complications: a meta-analysis. PLoS One 2013;8:e64926.

22. Chang YS, Fu HQ, Xiao YM, Liu JC. Nasogastric or nasojejunal feeding in predicted severe acute pancreatitis: a meta-analysis. Crit Care 2013;17:R118.

23. Al-Omran M, Albalawi ZH, Tashkandi MF, Al-Ansary LA. Enteral versus parenteral nutrition for acute pancreatitis. Cochrane Database Syst Rev 2010:CD002837.

24. Penn DE, Draganov PV, Wagh MS, Forsmark CE, Gupte AR, Chauhan SS. Prospective evaluation of the use of fully covered self-expanding metal stents for EUS-guided transmural drainage of pancreatic pseudocysts. Gastrointest Endosc 2012;76:679-684.

25. Bang JY, Varadarajulu S. Endoscopic ultrasound-guided management of pancreatic pseudocysts and walled-off necrosis. Clin Endosc 2014;47:429-431.

26. Antillon MR, Shah RJ, Stiegmann G, Chen YK. Single-step EUS-guided transmural drainage of simple and complicated pancreatic pseudocysts. Gastrointest Endosc 2006;63:797-803.

27. Park DH, Lee SS, Moon SH, et al. Endoscopic ultrasound-guided versus conventional transmural drainage for pancreatic pseudocysts: a prospective randomized trial. Endoscopy 2009;41:842-848.

28. Kahaleh M, Shami VM, Conaway MR, et al. Endoscopic ultrasound drainage of pancreatic pseudocyst: a prospective comparison with conventional endoscopic drainage. Endoscopy 2006;38:355-359.

29. Varadarajulu S, Christein JD, Tamhane A, Drelichman ER, Wilcox CM. Prospective randomized trial comparing EUS and EGD for transmural drainage of pancreatic pseudocysts (with videos). Gastrointest Endosc 2008;68:1102-1111.

30. Bang JY, Hawes R, Bartolucci A, Varadarajulu S. Efficacy of metal and plastic stents for transmural drainage of pancreatic fluid collections: a systematic review. Dig Endosc 2015;27:486-498.

31. Baron TH, Thaggard WG, Morgan DE, Stanley RJ. Endoscopic therapy for organized pancreatic necrosis. Gastroenterology 1996;111:755-764.

32. Seewald S, Groth S, Omar S, et al. Aggressive endoscopic therapy for pancreatic necrosis and pancreatic abscess: a new safe and effective treatment algorithm (videos). Gastrointest Endosc 2005;62:92-100.

33. Voermans RP, Besselink MG, Fockens P. Endoscopic management of walled-off pancreatic necrosis. J Hepatobiliary Pancreat Sci 2015;22:2026.

34. Seifert H, Biermer M, Schmitt W, et al. Transluminal endoscopic necrosectomy after acute pancreatitis: a multicentre study with long-term follow-up (the GEPARD Study). Gut 2009;58:1260-1266.

35. Walter D, Will U, Sanchez-Yague A, et al. A novel lumen-apposing metal stent for endoscopic ultrasound-guided drainage of pancreatic fluid collections: a prospective cohort study. Endoscopy 2015;47:63-67. 